\title{
ON PROJECTILES OF MINIMUM WAVE DRAG*
}

BY

\author{
WILLIAM R. SEARS \\ Cornell University**
}

1. Introduction. The wave resistance of slender bodies of revolution in symmetrical supersonic flow was calculated approximately by von Kármán, ${ }^{1}$ by means of a distribution of singularities along the axis of the projectile. The individual singularity is characterized by a potential of the form $\phi_{i}(x, r)=\left\{\left(x-\xi_{i}\right)^{2}-\alpha^{2} r^{2}\right\}^{-1 / 2}$, where $x, r$ are cylindrical coordinates, $x$ being measured downstream from the nose of the projectile and $r$ radially from the axis, $\xi_{i}$ is the value of $x$ corresponding to the singularity, $\alpha$ is the cotangent of the Mach angle of the undisturbed flow, so that

$$
\alpha=\sqrt{(U / a)^{2}-1},
$$

$U$ and $a$ being the stream velocity and the velocity of sound in the undisturbed flow. It will readily be verified that $\phi_{i}(x, r)$ is a solution of the linearized potential equation for supersonic flow with axial symmetry

$$
\left(\frac{U^{2}}{a^{2}}-1\right) \frac{\partial^{2} \phi}{\partial x^{2}}=\frac{\partial^{2} \phi}{\partial r^{2}}+\frac{1}{r} \frac{\partial \phi}{\partial r} .
$$

Von Kármán calculated the wave resistance by integrating the transport of momentum across a cylindrical surface enclosing the body. In his approximation, the integral is independent of $r$ and can be evaluated in the limit $r \rightarrow 0$. The result is $\dagger$

$$
R=-\pi \rho \int_{0}^{\infty} \int_{0}^{\infty} f^{\prime}(x) f^{\prime}(\xi) \log |x-\xi| d x d \xi,
$$

where $R$ is the wave resistance and $f(x)$ is the function specifying the distribution of singularities along the $x$ axis. For bodies of finite length $l, f(x)$ is found to be indentically zero for $x>l$; hence both integrals in (2) can be replaced by integrals from 0 to $l$.

For slender bodies, von Kármán showed that approximately

$$
f(x)=\frac{U}{2 \pi} \frac{d S}{d x},
$$

where $S$ is the cross-sectional area of the body.

In the present paper we shall amplify the analogy, already mentioned by von Kármán, between the wave resistance of a slender projectile and the induced drag of a wing. It will be shown that this analogy suggests a useful form for the calculation of

* Received June 11, 1946.

** This work was undertaken while the author was employed by Northrop Aircraft, Inc.

1 Th. de Kármán, The problem of resistance in compressible fluids, Atti del V Convegno della "Fondazione Alessandro Volta, ${ }^{n}$ Rome, 1935, pp. 222-276.

† Von Kármán'1, Eq. (9.12). It might be mentioned that this formula is most easily obtained from Eq. (9.11) of the same reference by first integrating by parts with respect to $x$ in order to obtain a form symmetrical in $x$ and $\xi$; it will then be found that a double integral carried over half the first quadrant of an $x, \xi$ plane can be identified with half the sume integral carried over the entire quadrant. 
the wave drag. The properties of projectiles of minimum wave drag for given length and volume, and for given length and caliber, will then be investigated.

2. The induced-drag analogy. Formula (2) for the wave drag can be written in the form

$$
R=-\pi \rho \int_{0}^{l} f^{\prime}(x) F(x) d x
$$

or, after integration by parts, assuming $f(0)=f(l)=0$; i.e. that the body has sharp points at front and rear,

$$
R=\pi \rho \int_{0}^{l} f(x) F^{\prime}(x) d x
$$

where

$$
F(x)=\int_{0}^{l} f^{\prime}(\xi) \log |x-\xi| d \xi .
$$

In the form $\left(2^{\prime \prime}\right)$, von Kármán's analogy between the wave resistance and the induced drag of a finite wing in the Prandtl lifting-line theory ${ }^{2}$ is evident: $f(x)$ is proportional to the circulation distribution over the span of the wing, $F^{\prime}(x)$ is the corresponding downwash distribution, and $R$ is the induced drag.

It is also useful to put (4) in another form, sometimes more convenient for calculation. Let us introduce the coordinates $\theta$ and $\vartheta$ defined by

$$
\begin{array}{ll}
x=\frac{l}{2}(1+\cos \theta), & 0 \leqq \theta, \\
\xi=\frac{l}{2}(1+\cos \vartheta), & \vartheta \leqq \pi .
\end{array}
$$

The expression for $F(x)$ then becomes

$$
F(x)=\frac{l}{2} \int_{0}^{\pi} f^{\prime}(\xi) \log |\cos \theta-\cos \vartheta| \sin \vartheta d \vartheta,
$$

provided that $\int_{0}^{l} f(\xi) d \xi=0$, as is always the case for closed bodies, in accordance with (3). Now the definitions in (5) can be taken to cover the range $-\pi \leqq \theta \leqq \pi,-\pi \leqq \vartheta \leqq \pi$, and $f^{\prime}(\xi)$ can arbitrarily be defined to be an odd function of $\vartheta$. Then (6) can easily be put into the form

$$
F(x)=\frac{l}{2} \int_{-\pi}^{\pi} f^{\prime}(\xi) \log \left|\sin \frac{\theta-\vartheta}{2}\right| \sin \vartheta d \vartheta
$$

or, after integration by parts,

$$
F(x)=-\frac{1}{2} \int_{-\pi}^{\pi} f(\xi) \cot \frac{\theta-\vartheta}{2} d \vartheta
$$

2L. Prandtl, Tragflitgeltheorie I, from Vier Abhandlungen zur Hydrodynamik and Aerodynamik, Göttingen, 1927, pp. 9-35. 
The induced-drag analogy pointed out above suggests that $f(\xi)$ be expanded in a sine series; this is the usual technique employed in the Prandtl wing theory: ${ }^{3}$

$$
f\left(\begin{array}{l}
x \\
\xi
\end{array}\right)=\frac{l U}{2} \sum_{1}^{N} b_{n} \sin \left(\begin{array}{c}
n \\
\vartheta \\
\vartheta
\end{array}\right) .
$$

Substituting in (7) and $\left(2^{\prime}\right)$, we obtain the following expression for the wave drag:

$$
R=\frac{\pi^{3}}{4} \frac{\rho U^{2}}{2} l^{2} \sum_{1}^{N} n b_{n}^{2}
$$

-again analogous to a well-known expression for the induced drag of a wing. ${ }^{3}$

3. Minimum wave drag for given volume and length. The expression for the crosssectional area $S$ corresponding to (8), in the approximation represented by (3), is

$$
S=\frac{\pi l^{2}}{4}\left\{\left[\pi-\theta+\frac{1}{2} \sin 2 \theta\right] b_{1}-\sum_{2}^{N} b_{n}\left[\frac{\sin (n-1) \theta}{n-1}-\frac{\sin (n+1) \theta}{n+1}\right]\right\} .
$$

It is clear that for closed projectiles, pointed front and rear, $b_{1}$ must vanish.* Also, the total volume occupied by the projectile is

$$
\text { Vol. }=\int_{0}^{l} S d x=\pi^{2}\left(\frac{l}{2}\right)^{3}\left(b_{1}-\frac{1}{2} b_{2}\right)
$$

or, for closed pointed bodies,

$$
\text { Vol. }=-\frac{1}{2} \pi^{2}\left(\frac{l}{2}\right)^{3} b_{2}
$$

Hence, for given length and volume, the minimum wave resistance is obtained when only $b_{2}$ is different from zero. The geometry of this body is given by

$$
S=-\frac{\pi l^{2} b_{2}}{4}\left(\sin \theta-\frac{1}{3} \sin 3 \theta\right)=-\frac{\pi l^{2} b_{2}}{3} \sin ^{3} \theta
$$

and its wave resistance is

$$
\begin{aligned}
R & =\frac{\pi^{3}}{2} \frac{\rho U^{2}}{2} l^{2} b_{2}^{2} \\
& =\frac{9}{8} \pi^{2} \frac{\rho U^{2}}{2} S_{\max }\left(\frac{d_{\max }}{l}\right)^{2} \\
& =\frac{8}{\pi} \frac{\rho U^{2}}{2}\left(\frac{l}{2}\right)^{2}\left[\frac{\text { Vol. }}{(l / 2)^{3}}\right]^{2} .
\end{aligned}
$$

This is eight times the wave drag of von Kármán's ogive* of equal length and volume, or about 11.1 times that of von Kármán's ogive of equal length and caliber. (It

${ }^{3}$ Th. von Kármán and J. M. Burgers, Aerodynamic Theory, edited by W.F. Durand, vol. 2, J. Springer, Berlin, 1934, pp. 172-175.

* If $b_{1} \neq 0$ while $b_{2}=b_{3}=\cdots=0$, the ogive considered by von Kármán ${ }^{1}$ is obtained. Its maximum cross-sectional area is $\pi^{2} l^{2} b_{1} / 4$ and occurs at its stern, $x=l$. According to (9), its wave drag is $\left(\pi^{3} / 4\right)\left(\rho U^{2} / 2\right) l^{2} b_{1}^{2}$ or $\left(\rho U^{2} / 2\right) S_{\max }\left(d_{\max } / l\right)^{2}$, where $S_{\max }$ is its maximum cross-sectional area and $d_{\max }$ is its maximum diameter, or caliber. 
should be mentioned that this comparison may be misleading in view of the fact that von Kármán's ogive has a blunt stern, so that its wave drag certainly does not represent its entire resistance, even in the absence of skin friction. Nevertheless, the wave resistance of that ogive may be taken as a convenient reference.)

The shape of the forward half of the symmetrical projectile represented in (13) is drawn in Fig. 1, for the case $l=4 d_{\max }$. For comparison, there is also shown the shape of von Kármán's ogive having the same caliber and one-half the length.

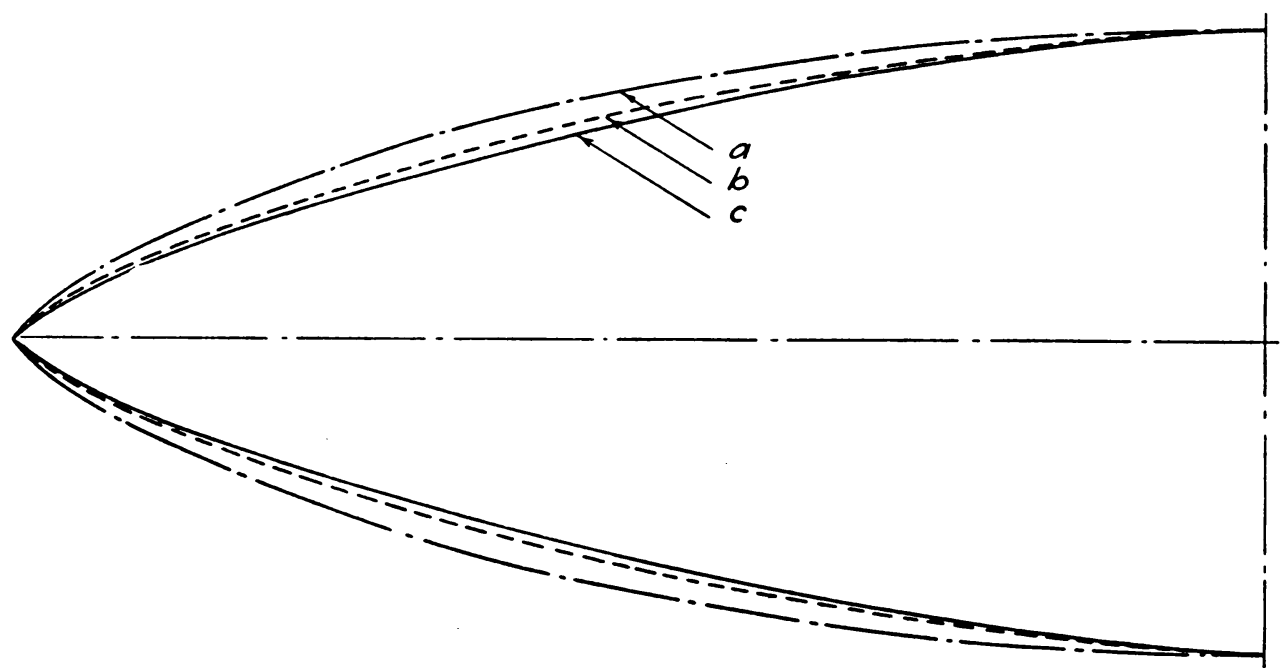

FIG. 1. Profiles of various projectiles of minimum wave drag: (a) volume and length given, (b) caliber and length given, (c) von Kármán's ogive of equal caliber and one-half the length. (Projectiles (a) and (b) are symmetrical fore-and-aft.)

4. Minimum wave drag for given caliber and length. To attack the problem of the body shape for minimum wave drag, caliber and length specified, we return to the expression for the wave drag given in $\left(2^{\prime}\right)$ and (4) and employ the methods of variation calculus. By virtue of the symmetry with respect to $x$ and $\xi$, the variation of the resistance with varying body form assumes a simple form; viz.,

$$
\begin{aligned}
\delta R= & -\pi \rho\left\{\int_{0}^{l} \delta f^{\prime}(x) \int_{0}^{l} f^{\prime}(\xi) \log |x-\xi| d \xi d x\right. \\
& \left.+\int_{0}^{l} f^{\prime}(x) \int_{0}^{l} \delta f^{\prime}(\xi) \log |x-\xi| d \xi d x\right\} \\
= & -2 \pi \rho \int_{0}^{l} \delta f^{\prime}(x) F(x) d x .
\end{aligned}
$$

In this section we shall provide for the possibility [excluded in obtaining $\left(2^{\prime \prime}\right)$ ] that $d S / d x$, and therefore $f(x)$, is discontinuous at the station where the maximum diameter occurs, $x=m$. Hence, integrating by parts in (15), and again assuning sharp points at bow and stern, we write 


$$
\begin{aligned}
\delta R & =-2 \pi \rho\left\{F(m) \delta[f(x)]_{m}-\int_{0}^{l} \delta f(x) F^{\prime}(x) d x\right\} \\
& =-2 \pi \rho\left\{F(m) \delta[f(x)]_{m}+\frac{U}{2 \pi} \int_{0}^{l} \delta S(x) F^{\prime \prime}(x) d x\right\},
\end{aligned}
$$

where $[f(x)]_{m}$ denotes the value of the discontinuity in $f(x)$ at $x=m$, and the area function $S(x)$ has been assumed to be continuous.

In the form (16) it is clear that the shape of the part of the body forward of the maximum section at $x=m$ can be held fixed while $S(x)$ is varied over the rear part to achieve a minimum of $R$; then the rear shape can be fixed in this minimum-drag configuration while $S(x)$ is varied in front to minimize $R$; the result will be the minimum-drag shape for given maximum cross section at $x=m$. We shall also assume that the discontinuity of slope represented by $[f(x)]_{m}$ is not varied in the process; it will appear later that this is valid. The minimum-drag condition $\delta R=0$ is then obtained when

$$
\begin{aligned}
& \left.\begin{array}{rl}
F^{\prime \prime}(x) & =0 \\
F(x) & =c_{1} x+c_{2}
\end{array}\right\} 0 \leqq x \leqq m, \\
& \left.\begin{array}{rl}
F^{\prime \prime}(x) & =0 \\
F(x) & =c_{3} x+c_{4}
\end{array}\right\} m \leqq x \leqq l .
\end{aligned}
$$

The analogy with the induced drag of a wing is again useful. The analogous problem is the following: to determine the spanwise circulation distribution $f(x)$ so as to obtain minimum induced drag, it being required that the total lift be zero, but that the lift carried on one side of a station $x=m$ have a given value, equal and opposite to that carried on the other side of that station. The result obtained in (17) states that the condition of minimum drag results when the downwash $F^{\prime}(x)$ is constant in each of the two parts of the wing.

Fortunately, investigations have been made $e^{4,5}$ of the behavior of the circulation distribution near a point on a lifting line where the downwash in discontinuous. It is found that the circulation function is continuous but has a vertical tangent and discontinuous curvature at such a point. Applying this result to our projectile problem, we can conclude that $f(x)$ will exhibit a singularity of this type at $x=m$. Moreover, since $F(x)$ can be interpreted as the downwash corresponding to the circulation distribution $S(x)$, we conclude that $F(x)$ cannot be discontinuous at $x=m$ if we exclude singularities of this type from the shape function $S(x)$. Accordingly, we write

$$
\left(c_{1}-c_{3}\right) m=c_{4}-c_{2}
$$

The resistance of the minimum-wave-drag body is easily calculated from $\left(2^{\prime}\right)$; it is

-A. Betz and E. Petersohn, Zur Theorie der Querruder, Z. angew. Math. Mech. 8, 253-257 (1928); also Nat. Advis. Com. for Aeron. Tech. Memo. No. 542 (1929).

- H. Multhopp, Die Berechnung der Auftriebsverteilung von Tragfïgeln, Luftfahrtforschung 15, 153169 (1938). 


$$
\begin{aligned}
R & =-\pi \rho\left\{\frac{U}{2 \pi}\left(c_{3}-c_{1}\right) S_{\max }+\left(c_{1} m+c_{2}\right)[f(x)]_{m}\right\} \\
& =\rho \frac{U}{2}\left(c_{1}-c_{3}\right) S_{\max },
\end{aligned}
$$

where $S_{\max }$ is the cross-sectional area at $x=m$.

The form of $f(x)$ corresponding to (17), and subsequently the shape of the body, can be determined by inverting (7) by means of the so-called "Reciprocity Theorem $":$

$$
f(x)=\frac{1}{2 \pi^{2}} \int_{0}^{2 \pi} F(\xi) \cot \frac{\theta-\vartheta}{2} d \vartheta .
$$

The quadratures involved are rather tedious, but can be carried out. The result is

$$
f(x)=\frac{1}{2 \pi^{2}}\left\{\left(c_{3}-c_{1}\right)(x-m) \log \frac{1-\cos (\theta+\mu)}{1-\cos (\theta-\mu)}+\left[\left(c_{3}-c_{1}\right) \mu+\pi c_{1}\right] l \sin \theta\right\},
$$

where $m=(l / 2)(1+\cos \mu)$. It can quickly be verified that this function has the type of singularity at $x=m$ that was predicted by the wing analogy.

This expression can be integrated again to evaluate the constants $c_{1}$ and $c_{3}$ and then to determine the function $S(x)$. By integrating from $x=0$ to $x=l$ it is determined that $\left(c_{3}-c_{1}\right)\left(\mu-\frac{1}{2} \sin 2 \mu\right)+\pi c_{1}=0$. Finally, by carrying out the lengthy quadratures necessary to apply the condition $(U / 2 \pi) S_{\max }=\int_{0}^{m} f(x) d x$, it is found that

$$
c_{1}=4 U S_{\max } \frac{\mu-\frac{1}{2} \sin 2 \mu}{l^{2} \sin ^{4} \mu} .
$$

The wave resistance (19) then assumes the form

or

$$
R=\frac{\rho U^{2}}{2} S_{\max }\left(\frac{d_{\max }}{l}\right)^{2} \frac{\pi^{2}}{\sin ^{4} \mu}
$$

$$
R=\frac{\rho U^{2}}{2} S_{\max }\left(\frac{d_{\max }}{l}\right)^{2}\left\{2 \frac{m}{l / 2}-\left(\frac{m}{l / 2}\right)^{2}\right\}^{-2} .
$$

Thus the wave drag varies symmetrically about $m=l / 2$ or $\mu=\pi / 2$, and is least if the maximum cross section is located at mid-length-i.e., for a symmetrical projectile. The wave drag of this projectile is $\pi^{2}$ times as great as that of von Kármán's ogive of equal length and caliber. Its shape is indicated in Figure 1.

5. Concluding remarks. A somewhat similar analysis of projectile shapes for minimum wave resistance has been made by Haack, ${ }^{7}$ who considered only symmetrical projectiles. The results obtained here are in agreement with Haack's for such projectiles, except for the value of the drag of the minimum-wave-drag body for given length and volume, which seems to have been tabulated erroneously in the earlier paper.

\footnotetext{
- R. Courant and D. Hilbert, Methoden der mathematischen Physik, vol. 1, J. Springer, Berlin, 1931, p. 83.

'W. Haack, Geschossformen kleinsten Wellenwiderstandes, Bericht 139 der Lilienthal-Gesellschaft für Luftfahrt.
} 